(Draft for Physical Review Fluids)

\title{
Scale-Locality of the Energy Cascade using Real Space Quantities
}

\author{
N. A. K. Doan, ${ }^{*}$ N. Swaminathan, and P. A. Davidson \\ Department of Engineering, University of Cambridge, \\ Cambridge CB2 1PZ, United Kingdom
}

\author{
M. Tanahashi \\ Department of Mechanical Engineering, \\ Tokyo Institute of Technology, 2-12-1 Ookayama, \\ Meguro-ku, Tokyo 152-8550, Japan
}

(Dated: July 13, 2018) 


\begin{abstract}
The classical energy cascade in turbulence as described by Richardson and Kolmogorov is predominantly a conjecture relying on the locality of interactions between scales of turbulence. This picture is generally accepted and assumes that energy and enstrophy transfers occur between neighbouring scales of turbulence and that vortex stretching plays a major role in the dynamics of this energy cascade. Direct numerical simulation data for $R e_{\lambda}$ ranging from 37 to 1131 is used to gather evidence for the cascade by investigating the energy and enstrophy fluxes between scales and the interplay between vorticity at one scale and strain at an adjacent scale. This is achieved by using a bandpass filter to educe the turbulent structures at various length scales allowing one to determine the fluxes between these scales and to interrogate the role of non-local (in physical-space) vortex stretching. It is shown that the structures of a length scale $L$ mostly transfer their energy to structures of size $0.3 L$ and that most of the enstrophy flux goes from structures of scale $L$ to $0.3 L$. Furthermore, vortical structures of a length scale $L_{\omega}$ are stretched mostly by straining structures of size 3 to $5 L_{\omega}$ and the stretching by eddies of sizes larger than $10 L_{\omega}$ is negligible. The stretching is dominated by the most extensive principal strain rate of the straining structures. These observations are found to be independent of $R e_{\lambda}$ for the range investigated in this study. These results provide strong evidence for the classical view of an energy cascade transferring energy from large to small scales through a hierarchy of steps, each step consisting of the stretching of vortices by somewhat larger structures.
\end{abstract}

\footnotetext{
*nakd2@cam.ac.uk
} 


\section{INTRODUCTION}

Turbulent flows are often pictured as a hierarchy of eddies of different scales whose morphology and spatial clustering depend on the turbulence Reynolds number. Many views have been proposed in the past to describe the interaction of these eddies and how the energy cascades through these scales from the large energy-containing eddies to the small dissipative structures. In particular, the Richardson/Kolmogorov energy cascade [1,2] conjectured that kinetic energy introduced at large scales is progressively transferred to smaller and smaller scales through the inertial range, eventually reaching the Kolmogorov scale where it is dissipated by the fluid viscosity. The key assumption of this conjecture is the scale locality of the cascade, the idea by which eddies mainly interact with and transfer energy to eddies of neighbouring (smaller) sizes.

Historically, turbulence and turbulent structures have often been described in spectral space and their wave numbers become a surrogate for their physical scales. In this formalism, the locality of the energy cascade is understood as the proximity between interacting wave numbers. From this perspective, various closure models relying on this notion of cascade for turbulence were developed based on algebraic expressions for the spectral kinetic energy transfer function [3-5] with reasonable success. Furthermore, the locality of the energy cascade in wave number space was assessed by studying the interactions between triads of wave numbers and their contribution to the energy flux. It was shown that the energy transfer was dominated by local wave number triad interactions [6-9]. Approaches analogous to the analysis of the spectral energy transport equation had also been performed in physical space using the two-point velocity correlation transport equation and the Karman-Howarth equation [10]. In this context, the family of closures proposed are the quasi-normal-type schemes which assume that for the fourth-order velocity correlations terms, the joint-pdf of the velocity field measured at two points is Gaussian. This then allows to close the KarmanHowarth equations and these closures are reviewed in [11]. However, there is an inherent arbitrary nature to the approximations used and the heuristic modifications required to correct these models. Thus, there is no consensus on the locality of the scale interactions in

physical space. Indeed, some studies have both supported the assumption of scale-locality [12-15] while others have shown that energy could be transferred directly from large scales to much smaller ones [16-18]. Clear evidence for this scale-locality of the energy cascade 
using real space quantities is scant.

Nonetheless, from the physical space perspective, since Taylor's work [19, 20], it has often been suggested that the mode of energy transfer across the scales is through vortex stretching, in which vortical structures of a given scale are stretched and intensified by larger vortices, leading to the transfer of energy from the larger to the smaller eddies with the smallest eddies having a worm-like shape, an idea that dates back to Burgers [21]. In particular, the rate of generation of enstrophy, $\Omega=|\boldsymbol{\omega}|^{2} / 2$, by vortex stretching, $\psi=\omega_{i} \omega_{j} S_{i j}$, is often taken as a proxy for energy transfer in real scale space [22, 23].

Despite the relatively wide acceptance of this classical picture interweaving energy cascade and vortex stretching, rigorous evidence supporting this association remains elusive and additional investigations are required to support the vortex stretching picture and its locality in scale space. Indeed, past Direct Numerical Simulations (DNS) [24-27] and experiments $[28,29]$ show that the vorticity aligns preferentially with the intermediate strain rate, $\beta$, which suggests the formation of vortex sheets rather than vortex tubes [22]. By contrast, one would expect $\boldsymbol{\omega}$ to preferentially align with $\alpha$ if the vortices being stretched are tubelike (worms) [22]. This apparent disconnect stems from the influence of local (in physical space) straining associated with the self-induced strain fields of vortices [30, 31]. Indeed, when these effects are excluded and only non-local straining in physical space retained, it is found that $\boldsymbol{\omega}$ is indeed aligned, on average, with $\alpha$ [32-34]. The effects of self-straining can be filtered out by using a bandpass filter to educe vorticity and straining structures of different scales and examine their mutual interaction. This shows that, for modest values of Taylor microscale Reynolds numbers, $R e_{\lambda}$, a vortex of a given size is stretched primarily by the $\alpha$ strain from structures that are 3 to 5 times larger than the vortical structure [34]. These results were obtained for homogeneous isotropic turbulence and similar results supporting the scale-locality of the energy cascade and the vortex stretching in shear flows were reported in [35-37]. By contrast, if one does only consider the alignment between vorticity and straining structures at the same scale, and thus not excluding the self-straining effect, the preferential alignment of $\boldsymbol{\omega}$ with $\beta$ is retained $[34,38]$.

The relatively low $R e_{\lambda}$ of 107 [32] and 141 [34] in the previous studies raises questions as to the validity of their findings for higher Reynolds numbers, particularly as it is often suggested that high $R e_{\lambda}$ turbulence presents dynamical features that differ from lower Reynolds number turbulence. For example, Ishihara et al. [39] examined data at $R e_{\lambda}=1131$ and highlighted 
the appearance of thin shear layers consisting of clusters of thin intense vortex tubes. They suggested that the spatial structure of turbulence undergoes a transition as $R e_{\lambda}$ approaches 1000 , with larger values of $R e_{\lambda}$ favouring the clustering of vortex worms into slabs or sheets. This clustering is less apparent at lower $R e_{\lambda}$. Thus, a definitive theory of the energy cascade, applicable across all $R e_{\lambda}$, remains elusive.

The objectives of this study are (i) to revisit the classical cartoon of the energy cascade using DNS data to investigate its locality in scale space, through a clear analysis of the energy and enstrophy transfers, and vortex stretching mechanism between various scales; (ii) to investigate whether or not the nature of the cascade changes with $R e_{\lambda}$ and in particular whether the results observed in [34] for $R e_{\lambda}=141$ are valid for smaller and larger Reynolds numbers; and (iii) to study the morphology of the straining and strained structures. These objectives are addressed by analysing DNS data for $R e_{\lambda}$ ranging from 37 to 1131 . Each dataset is analysed using the bandpass filtering method [34] which allows one to focus on structures of a chosen length scale, $L$. The morphological features of these structures are then analysed using Minkowski functionals. The present work will thus mainly focus on the scale-locality of the energy cascade using quantities in real space rather than in wave number space and not on the spatial locality of the energy/enstrophy transfer.

The scale decomposition framework used here is described in section II along with the methodologies used for analysis. Details on the various DNS datasets used are provided in section III. The results are then discussed in section IV which focuses on (i) the morphological features of turbulent structures, (ii) the transfer of energy and enstrophy across scales of turbulence and (iii) the interaction of turbulent structures through the analysis of the vortex stretching mechanism. Conclusions are summarised in the final section.

\section{ANALYSIS METHOD}

\section{A. Scale decomposition}

To analyse the scale-by-scale transfer and interaction, following earlier works [40, 41], one can decompose the velocity, $\boldsymbol{u}$, and vorticity, $\boldsymbol{\omega}$, into large and small scales such that $\boldsymbol{u}=\boldsymbol{u}^{\mathbb{L}}+\boldsymbol{u}^{\mathbb{S}}$ and $\boldsymbol{\omega}=\boldsymbol{\omega}^{\mathbb{L}}+\boldsymbol{\omega}^{\mathbb{S}}$, where the superscripts $\mathbb{L}$ and $\mathbb{S}$ denote the contribution of structures respectively larger and smaller than a specified scale $r$. Such decomposition is 
not unique and depends on the low-pass filter used to yield $\boldsymbol{\omega}^{\mathbb{L}}$ and $\boldsymbol{u}^{\mathbb{L}}$. This aspect will be tackled subsequently.

Nonetheless, using this formalism, an energy equation for the large and small scales can be deduced by taking the dot product of $\boldsymbol{u}^{\mathbb{L}}$ and $\boldsymbol{u}^{\mathbb{S}}$ with the Navier-Stokes equations and ensemble averaging the resulting equations [40,41]. This gives

$$
\begin{gathered}
\frac{\partial}{\partial t}\left\langle\frac{1}{2}\left(\boldsymbol{u}^{\mathbb{L}}\right)^{2}\right\rangle=-\Pi_{V}-\nu\left\langle\left(\boldsymbol{\omega}^{\mathbb{L}}\right)^{2}\right\rangle \\
\frac{\partial}{\partial t}\left\langle\frac{1}{2}\left(\boldsymbol{u}^{\mathbb{S}}\right)^{2}\right\rangle=\Pi_{V}-\nu\left\langle\left(\boldsymbol{\omega}^{\mathbb{S}}\right)^{2}\right\rangle
\end{gathered}
$$

for the energy in scales $\mathbb{L}$ and $\mathbb{S}$ respectively. The symbol $\Pi_{V}$ is defined as:

$$
\Pi_{V}(r)=\left\langle S_{i j}^{\mathbb{L}} \tau_{i j}^{\mathbb{S}}-S_{i j}^{\mathbb{S}} \tau_{i j}^{\mathbb{L}}\right\rangle
$$

with $S_{i j}$ the symmetric strain-rate tensor, $\tau_{i j}^{\mathbb{L}}=-u_{i}^{\mathbb{L}} u_{j}^{\mathbb{L}}$ and $\tau_{i j}^{\mathbb{S}}=-u_{i}^{\mathbb{S}} u_{j}^{\mathbb{S}}$ are the Reynolds stresses at large and small scales. In the present formalism, as $\Pi_{V}(r)$ appears in both equations with an opposite sign, it can be interpreted as the flux of energy from larger to smaller scales across the scale $r$.

In a similar manner, by taking the dot product of $\boldsymbol{\omega}^{\mathbb{L}}$ or $\boldsymbol{\omega}^{\mathbb{S}}$ with the vorticity equation, one can obtain the enstrophy equations for the large and small structures [41]:

$$
\begin{gathered}
\frac{\partial}{\partial t}\left\langle\frac{1}{2}\left(\boldsymbol{\omega}^{\mathbb{L}}\right)^{2}\right\rangle=-F(r)+G^{\mathbb{L}}(r)-\nu\left\langle\left(\nabla \times \boldsymbol{\omega}^{\mathbb{L}}\right)^{2}\right\rangle \\
\frac{\partial}{\partial t}\left\langle\frac{1}{2}\left(\boldsymbol{\omega}^{\mathbb{S}}\right)^{2}\right\rangle=F(r)+G^{\mathbb{S}}(r)-\nu\left\langle\left(\nabla \times \boldsymbol{\omega}^{\mathbb{S}}\right)^{2}\right\rangle
\end{gathered}
$$

with

$$
\begin{gathered}
F(r)=\left\langle\boldsymbol{\omega}^{\mathbb{L}} \cdot\left(\boldsymbol{u} \cdot \nabla \boldsymbol{\omega}^{\mathbb{S}}\right)\right\rangle=-\left\langle\boldsymbol{\omega}^{\mathbb{S}} \cdot\left(\boldsymbol{u} \cdot \nabla \boldsymbol{\omega}^{\mathbb{L}}\right)\right\rangle \\
G^{\mathbb{L}}(r)=\left\langle\boldsymbol{\omega}^{\mathbb{L}} \cdot(\boldsymbol{\omega} \cdot \nabla \boldsymbol{u})\right\rangle=\left\langle\omega_{i}^{\mathbb{L}} \omega_{j} S_{i j}\right\rangle \\
G^{\mathbb{S}}(r)=\left\langle\boldsymbol{\omega}^{\mathbb{S}} \cdot(\boldsymbol{\omega} \cdot \nabla \boldsymbol{u})\right\rangle=\left\langle\omega_{i}^{\mathbb{S}} \omega_{j} S_{i j}\right\rangle
\end{gathered}
$$

Here, $G^{\mathbb{L}}(r)$ and $G^{\mathbb{S}}(r)$ represent the generation of enstrophy via vortex stretching at large and small scales respectively and $F(r)$ is the transfer or flux of enstrophy across the scale $r$, from larger to smaller scales.

From this formalism, the transfer of energy and enstrophy from scales $\mathbb{L}$ to $\mathbb{S}$ across the scale $r$ can be analysed by studying the flux functions, $\Pi_{V}$ for the energy and $F$ for the 
enstrophy as has been done by Davidson et al. [41]. The interest of the present work is to investigate the interactions between a given scale $L$ and various smaller scales $S$ to identify the range of scales interacting with structures at scale $L$ and thus assess the locality of the energy/enstrophy transfer. This is achieved by using the bandpass filtering method proposed by Leung et al. [34] described in the next section.

\section{B. Bandpass filtering}

This filtering procedure allows one to educe structures of a chosen length scale, $L$, in physical space as has been demonstrated by Leung et al. [34]. In this method, the Fourier transform of the bandpass filtered velocity field is simply related to the Fourier transform of the unfiltered field and it is written as

$$
\widehat{\boldsymbol{u}}_{b}^{L}=\frac{\alpha}{\sqrt{L}} 2 \kappa^{2} \exp \left(-\kappa^{2}\right) \widehat{\boldsymbol{u}}(\boldsymbol{k})
$$

where $\boldsymbol{k}$ is the wave number vector, $\kappa=k L / 2$ with $k=|\boldsymbol{k}|$ and $\alpha=\sqrt{2}$ [34]. Then, applying the inverse Fourier transform to the filtered field, a bandpass filtered velocity field, $\boldsymbol{u}_{b}^{L}$, is obtained. The fields of vorticity, strain-rate and Reynolds stresses at scale $L$ are then computed through:

$$
\boldsymbol{\omega}^{L}=\nabla \times \boldsymbol{u}_{b}^{L}, \quad S_{i j}^{L}=\frac{1}{2}\left(\frac{\partial u_{b, i}^{L}}{\partial x_{j}}+\frac{\partial u_{b, j}^{L}}{\partial x_{i}}\right), \quad \tau_{i j}^{L}=-u_{i}^{L} u_{j}^{L}
$$

By using this technique, one can educe these "large" and "small" scales separately, and not necessarily across a given scale $r$. The bandpass filtered velocity at some specified scales $L$ and $S$, with $L>S$, can be used in Eqs. (3) and (6) to directly compute the transfer of energy or enstrophy from structures of scale $L$ to those of scale $S$, ie,

$$
\begin{gathered}
\Pi_{V, b}^{L \rightarrow S}=\left\langle S_{i j, b}^{L} \tau_{i j, b}^{S}-S_{i j, b}^{S} \tau_{i j, b}^{L}\right\rangle \\
F_{b}^{L \rightarrow S}=\left\langle\boldsymbol{\omega}_{b}^{L} \cdot\left(\boldsymbol{u} \cdot \nabla \boldsymbol{\omega}_{b}^{S}\right)\right\rangle
\end{gathered}
$$

where the subscript $b$ indicates the use of bandpass filtered fields.

Furthermore, to analyse the vortex stretching mechanism, one can analyse the interplay between the vorticity at scale $L_{\omega}$ and the strain rate at $L_{s}$. Indeed, the rate of generation of enstrophy at scale $L_{\omega}$ due to straining structures at scale $L_{s}$ can be expressed as:

$$
\psi=\omega_{i}^{L_{\omega}} \omega_{j}^{L_{\omega}} S_{i j}^{L_{s}}=\left|\omega^{L_{\omega}}\right|^{2}\left(\alpha^{L_{s}} \cos ^{2} \theta_{\alpha}+\beta^{L_{s}} \cos ^{2} \theta_{\beta}+\gamma^{L_{s}} \cos ^{2} \theta_{\gamma}\right)
$$


where $\alpha^{L_{s}}, \beta^{L_{s}}$ and $\gamma^{L_{s}}$ are the principal components of $S_{i j}^{L_{s}}$ with $\alpha^{L_{s}}>\beta^{L_{s}}>\gamma^{L_{s}}$ and $\theta_{i}$ are the corresponding angles between the vorticity vector and these principal components. Thus, to analyse the vortex stretching mechanism, one can analyse the alignment statistics as measured by the pdf of $\cos \left(\theta_{i}\right)$ between vortical structures at a length scale $L_{\omega}$ and straining structures at a larger length scale $L_{s}$.

\section{Morphology descriptor}

The Minkowski functionals are used to perform an objective analysis of the morphology of turbulent structures educed at various scales using the bandpass filtering methodology. These Galilean invariants are commonly used in cosmology, for example [42], and describe the morphology of a given three dimensional structure. The four functionals for a three dimensional structure are given by [43]:

$$
V_{0}=V, \quad V_{1}=\frac{A}{6}, \quad V_{2}=\frac{1}{3 \pi} \int_{A} \frac{\mathcal{K}_{1}+\mathcal{K}_{2}}{2} d A, \quad V_{3}=\frac{1}{2 \pi} \int_{A} \mathcal{K}_{1} \mathcal{K}_{2} d A
$$

$V$ is the volume enclosed by the three dimensional object with a surface area, $A$. The principal curvatures at a given point on this surface are $\mathcal{K}_{1}$ and $\mathcal{K}_{2}$. Using these Minkowski functional, one can then define the shapefinders - length, $\ell$, width, $w$, and thickness, $t$, ordered as $t<w<\ell$ and computed using

$$
t=\frac{V_{0}}{2 V_{1}}, \quad w=\frac{2 V_{1}}{\pi V_{2}}, \quad \ell=\frac{3 V_{2}}{2 V_{3}}
$$

It should be noted that particular care is taken with $V_{3}$ as holes could be present in the structure, thus yielding $V_{3} \leq 0$. In those case, $\ell$ is defined as $\ell=3 V_{2} /(4(G+1))$ where $G=1-V_{3} / 2$ is the genus of the structure. The genus of a structure is the number of cuts that can be made along a simple curve on the object without splitting it [44]. From these three characteristics length scales, two quantities called planarity, $\mathcal{P}$, and filamentarity, $\mathcal{F}$, can be defined [43]:

$$
\mathcal{P}=\frac{w-t}{w+t}, \quad \mathcal{F}=\frac{\ell-w}{\ell+w}
$$

These two dimensionless quantities are bounded between 0 and 1 and can then be used to classify the considered three dimensional object in terms of simple shapes, such as a blob or sphere $(\mathcal{P}, \mathcal{F})=(0,0)$, very long tube $(0,1)$, thin sheet $(1,0)$ and very long ribbon $(1,1)$ as noted by Leung et al. [34]. 
This procedure has been applied on vortical and straining structures educed at various length scales as will be detailed in section IV A.

\section{DNS DATASET}

DNS data of homogeneous isotropic turbulence are used here. These datasets, from Ishihara et al. [39], Tanahashi et al. [45], Donzis et al. [46], Kobayashi et al. [47], cover $\operatorname{Re}_{\lambda}$ from 37 to 1131 (or integral length scale Reynolds number, $R e_{l}$, from 97 to 36,345) as listed in Table I. These datasets cover the widest range of Reynolds number currently available and are from a variety of research groups. The computational domain for each dataset is a triply periodic cube of length $2 \pi$ with $N$ grid points in each direction. Two cases at $R e_{\lambda}=140$ and 1131 have forced turbulence while all the others have freely decaying turbulence. The case at $R e_{\lambda}=140$ uses stochastic forcing at large scale, applied on wave numbers smaller than $K_{c}=2 \sqrt{2}[46,48]$. For the case at $R e_{\lambda}=1131$, the forcing is performed in wave-number

space as $\widehat{\boldsymbol{f}}(\boldsymbol{k})=c \widehat{\boldsymbol{u}}(\boldsymbol{k})$ where $\widehat{\boldsymbol{f}}$ is the Fourier transform of the forcing and $c$ is a non-zero coefficient independent of $k$ and is equal to $\Gamma$ for $k<K_{C}$ and 0 otherwise. The value $\Gamma$ was adapted at each time step so as to maintain the total kinetic energy, $E$, inside the domain. The value of $K_{c}$ was taken to be 2.5 for the $R e_{\lambda}=1131$ case [39]. It should be noted that these forcing schemes mainly affect structures of turbulence of a scale of about $2 \pi / K_{c}$ and have a decreasing influence as one considers smaller and smaller scales. This scale of $2 \pi / K_{c}$ corresponds to approximately $190 \eta$ and $4900 \eta$ respectively for the cases at $R e_{\lambda}=140$ and $R e_{\lambda}=1131$, where $\eta$ is the Kolmogorov length scale. Thus for the range of scales considered here, the influence of the forcing scheme should be limited. This will be further discussed in Sec. IV B.

The analysis presented here is performed on snapshots of the data taken once the turbulence is fully developed, as judged by the velocity derivative skewness approaching -0.5. Since only instantaneous snapshots are available for analysis, the temporal aspects of the energy cascade is not studied here. Characteristics of each case required for this study are summarised in Table I and more details on these datasets can be found in the relevant references. 


\begin{tabular}{ccccccc}
\hline$R e_{\lambda}$ & $N$ & $l / \eta$ & $\lambda / \eta$ & Forced or Decaying Reference & Institution \\
\hline 37.1 & 128 & 31 & 11.8 & $\mathrm{D}$ & {$[45]$} & Tokyo Tech \\
64.9 & 128 & 55 & 17.1 & $\mathrm{D}$ & {$[45]$} & Tokyo Tech \\
97.1 & 256 & 100 & 20.9 & $\mathrm{D}$ & {$[45]$} & Tokyo Tech \\
140 & 256 & 101 & 28.0 & $\mathrm{~F}$ & {$[46]$} & Georgia Tech \\
141.1 & 400 & 200 & 24.0 & $\mathrm{D}$ & {$[45]$} & Tokyo Tech \\
222.7 & 640 & 494 & 29.3 & $\mathrm{D}$ & {$[47]$} & Tokyo Tech \\
393.8 & 1536 & 1146 & 39.0 & $\mathrm{D}$ & Unpublis. & Tokyo Tech \\
1131 & 4096 & 2137 & 66.5 & $\mathrm{~F}$ & {$[39]$} & Nagoya Univ \\
\hline
\end{tabular}

TABLE I. Characteristics of the (forced or decaying) homogeneous isotropic turbulence DNS database. $l$ is the integral length scale, $\lambda$ the Taylor microscale and $\eta$ the Kolmogorov length scale.

\section{RESULTS}

\section{A. Morphology of the turbulent vortices}

The turbulent velocity fields have been obtained at various scales by using the bandpass filter with length scale, $L$, varying from $5 \eta$ to up to $1300 \eta$ depending on the dataset. From these fields, the strained (enstrophy) and straining structures can be deduced using Eq. (10). Figure 1 shows examples of the strained and straining structures for $R e_{\lambda}=140$ and 1131 . The length scales $L_{s}$ and $L_{\omega}$ are respectively $24 \eta$ and $5 \eta$ for Figs. 1a and 1c. These length scales are $L_{s}=75 \eta$ and $L_{\omega}=24 \eta$ for Fig. $1 \mathrm{~b}$, and they are $L_{s}=750 \eta$ and $L_{\omega}=150 \eta$ for Fig. 1d. These specific combinations of $L_{s}$ and $L_{\omega}$ are chosen based on the suggestions of Leung et al. [34] which showed that most stretching imparted on strained structures was coming from straining structures 3 to 5 times larger. The isosurfaces are thresholded at a value of $\mu+2 \sigma$, where $\mu$ is the mean and $\sigma$ is the standard deviation. This threshold was chosen following the work of Leung et al. [34] to focus on regions with intense vorticity or strain rate. Despite the variation in the volume of these structures, it is observed that their general morphology is not significantly modified by varying the threshold, as also observed by Leung et al. [34]. Indeed other thresholds of $\mu+\sigma$ and $\mu+3 \sigma$ yielded similar results (not shown here for brevity). The enstrophy and straining structures are more space filling in 
the larger $R e_{\lambda}$ case, irrespective of their scales. This was assessed by computing the volume fraction occupied by these structures at various Reynolds numbers. For example, when comparing the enstrophy structures of Fig. 1a to 1c, they occupy respectively $2.97 \%$ and $10.4 \%$ for cases $R e_{\lambda}=140$ and $R e_{\lambda}=1131$. Similarly, the straining structures of Fig. 1a for $R e_{\lambda}=140$ only occupy $4.3 \%$ of the volume compared to $24.64 \%$ for $R e_{\lambda}=1131$ shown in Fig. 1c. Furthermore, the enstrophy structures are predominantly tube and blob-like with large strain fields at their periphery, as in the Burgers vortex model. This is illustrated in Fig. 2 where a single enstrophy structure has been isolated from Fig. 1c along with its neighbouring straining structures.

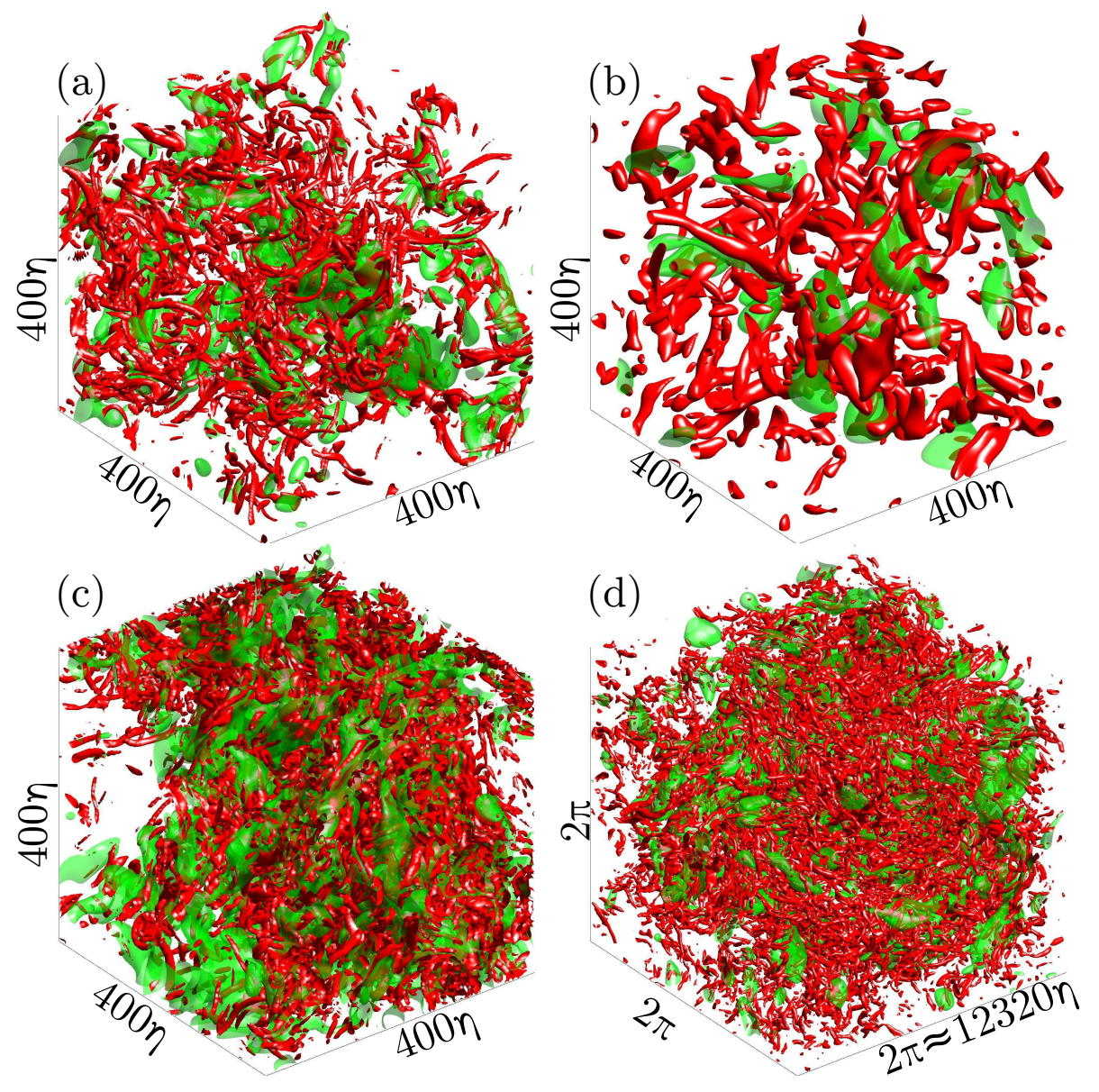

FIG. 1. Iso-surfaces of enstrophy (red) and straining (green) structures with a threshold value of $\mu+2 \sigma$, where $\mu$ is the mean and $\sigma$ is the rms. Panels (a) \& (b) are for $R e_{\lambda}=140$ and (c) \& (d) are for $R e_{\lambda}=1131$. In (a) and (c) $L_{s}=24 \eta$ and $L_{\omega}=5 \eta$, in (b) $L_{s}=75 \eta$ and $L_{\omega}=24 \eta$ and in (d) $L_{s}=750 \eta$ and $L_{\omega}=150 \eta$.

The shapefinders, $\mathcal{P}$ and $\mathcal{F}$, for the strained and straining structures seen in Fig. 1 are 

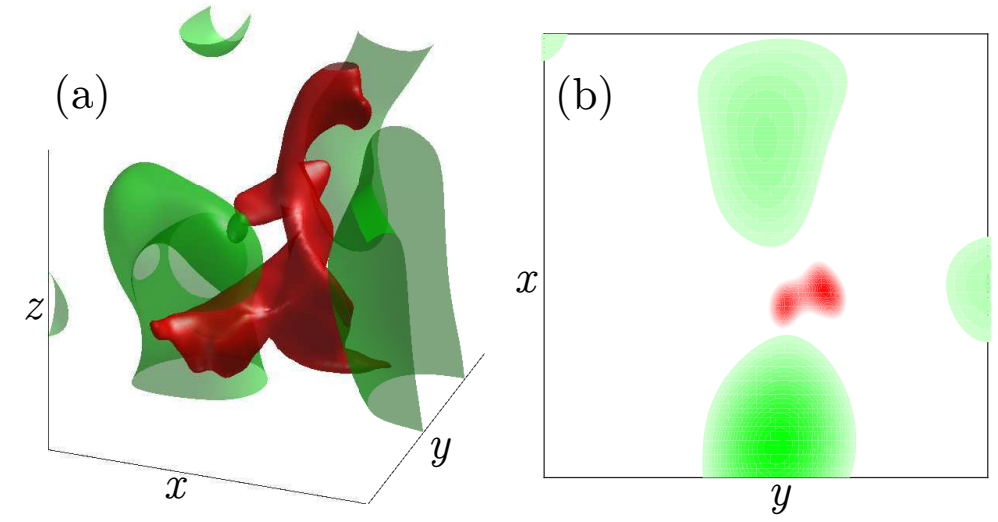

FIG. 2. (a) Isolated single iso-surface of enstrophy (red) and dissipation (green) structures with a threshold value of $\mu+2 \sigma$ extracted from the case $R e=1131$. (b) The associated mid $x-y$ plane distribution.

plotted in Fig. 3. The results are shown as contours of joint probability density function (pdf) of $\mathcal{P}$ and $\mathcal{F}$ for the strained structures and as scatter plots for the straining structures. This particular choice is due to the small number of straining structures at larger scales preventing the computation of a smooth pdf. For example, there are only 18 straining structures observed in Fig. $1 \mathrm{~b}$ for $L_{s}=75 \eta$ and their $\mathcal{P}$ and $\mathcal{F}$ are shown as scatter plot in Fig. 3b. The ranges of $\mathcal{P}$ and $\mathcal{F}$ observed in Fig. 3 suggest that the educed structures seen in Fig. 1 are mostly blob-like (low $\mathcal{P}$ and low $\mathcal{F}$ ) or tube-like (low $\mathcal{P}$ and medium or high $\mathcal{F}$ ) even for the largest $R e_{\lambda}$ and $L_{\omega}=150 \eta$ considered here. More specifically, the straining structures tend to have a blob-like aspect ratio for all length scales considered here, while the small scale vortices with $L_{\omega}=5 \eta$ present a tube-like morphology (see Figs. 3a and 3c). However, as $L_{\omega}$ increases, the enstrophy structures starts to show blob-like morphology as observed by the shift in the position of the joint-pdf towards lower value of $\mathcal{F}$ (compare Fig. 3a to 3b, and Fig. 3c to 3d). Similar observations about the difference in the shapes of the vortical and straining structures were also made in [34] using bandpass filters and in [49] based on the fractal dimensions for the intense vortical and straining structures.

\section{B. Energy and enstrophy transfer}

The normalised energy transfer function, $\widehat{\Pi}_{V, b}^{L \rightarrow S}=\prod_{V, b}^{L \rightarrow S} / \max \left(\Pi_{V, b}^{L \rightarrow S}\right)$, is shown in Fig. 4 where it is plotted for three different Reynolds numbers $R e_{\lambda}=97.1,222.7$ and 1131. For 

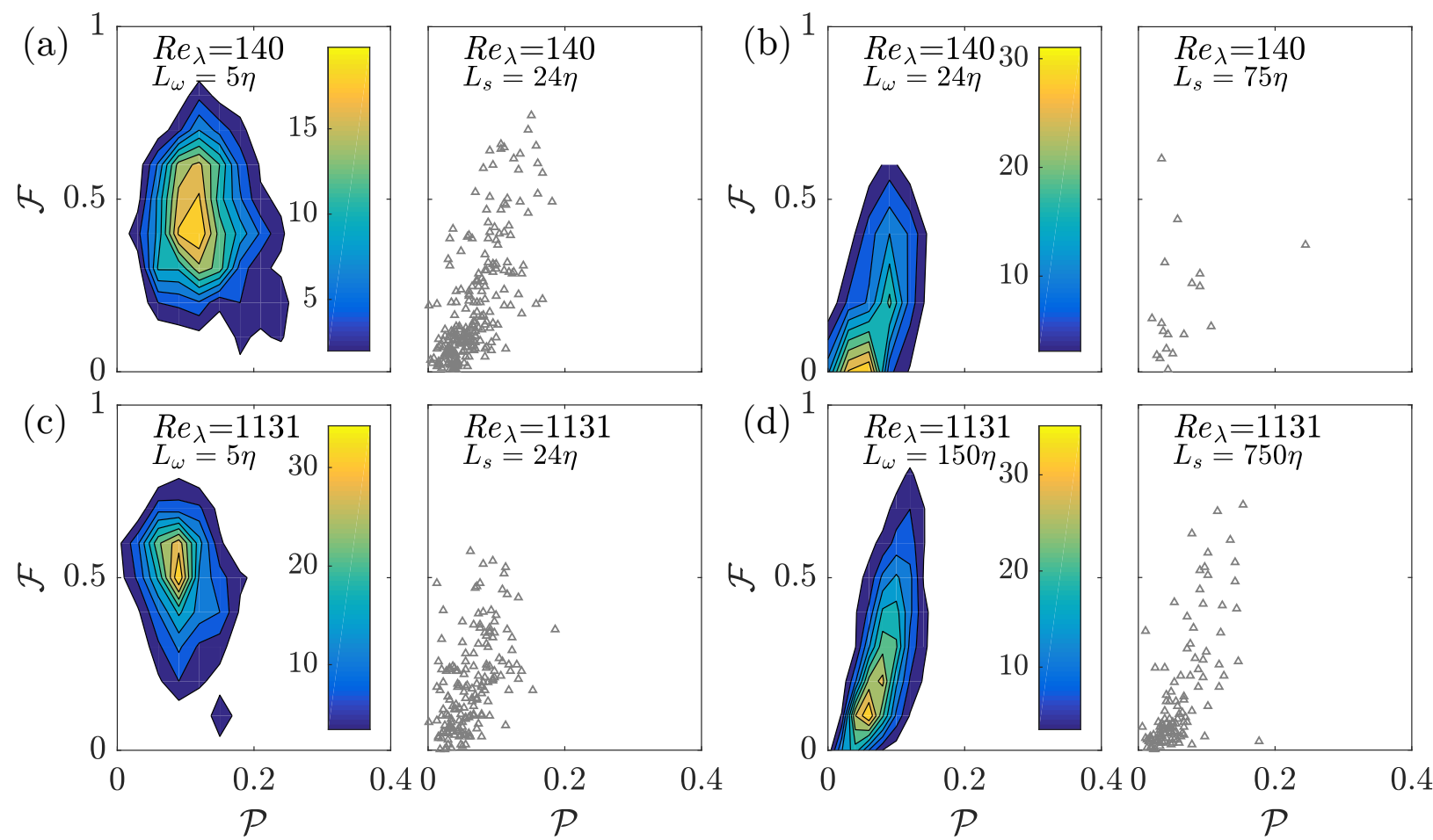

FIG. 3. Joint PDF of planarity, $\mathcal{P}$, and filamentarity, $\mathcal{F}$ of the strained (or enstrophy) structures seen in Fig. 1.

each case, a large scale, $L$, is picked first and the small scale $S$ is varied to determine which small scale receives the most energy from the structures of size $L$. This can be repeated for various values of $L$ to analyse if the range of scale interactions evolves across scales of turbulence in a particular fashion or not.
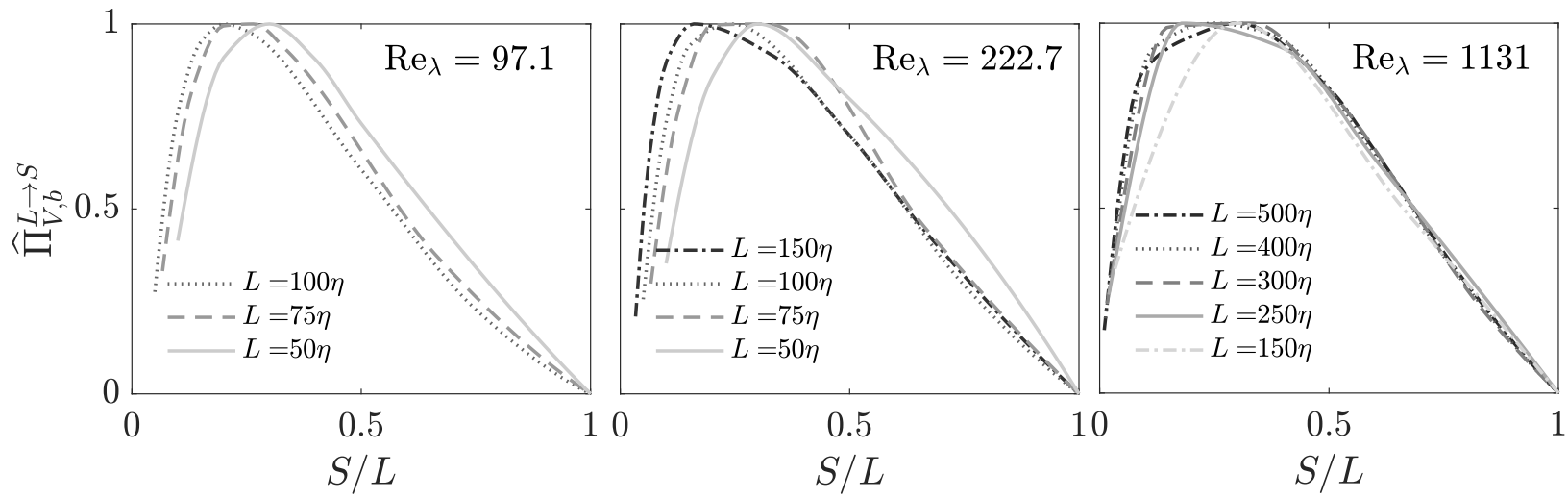

FIG. 4. Normalised energy transfer function, $\widehat{\Pi}_{V, b}^{L \rightarrow S}$, for various cases.

It is observed that $\widehat{\Pi}_{V, b}^{L \rightarrow S}$ peaks for values of $S / L$ around 0.3 for all values of $L$. This 
indicates that structures at scale $L$ mostly transfer energy to structures having a size of about $\sim 0.3 L$. Furthermore, the normalising quantity, $\max \left(\prod_{V, b}^{L \rightarrow S}\right)$, increases with decreasing $L$, for the range of $L$ studied here, and increases with increasing $R e_{\lambda}$. As this analysis is performed for various $L$, the results in Fig. 4 show that there exists a locality in the energy transfer for the range of scales across the turbulence spectrum. This strongly supports the energy cascade picture which portrays that the energy is transferred from one scale to its neighbouring smaller scale. Furthermore, this result is observed to be insensitive for all the Reynolds numbers considered here suggesting that the energy cascade picture is robust and does not change with $R e_{\lambda}$.

A similar analysis is performed for the enstrophy flux between two scales, $L$ and $S$. The normalised value of the enstrophy flux, $\widehat{F}_{b}^{L \rightarrow S}=F_{b}^{L \rightarrow S} / \max \left(F_{b}^{L \rightarrow S}\right)$, is shown in Fig. 5 for the same cases considered for the energy transfer in Fig. 4. The behaviour of $\widehat{F}_{b}^{L \rightarrow S}$ is very similar to that for the energy transfer. The enstrophy of scale $L$ is mostly transferred to scales of about $0.3 L$ for all $L$ considered and $\max \left(F_{b}^{L \rightarrow S}\right)$ has the same behaviour as $\max \left(\Pi_{V, b}^{L \rightarrow S}\right)$ with variations of $R e_{\lambda}$ and $L$. This similar behaviour between $\widehat{F}_{b}^{L \rightarrow S}$ and $\widehat{\Pi}_{V, b}^{L \rightarrow S}$ hints at a close relation between the energy and enstrophy transfers and the enstrophy still cascades from large to small scales just like the energy [41].
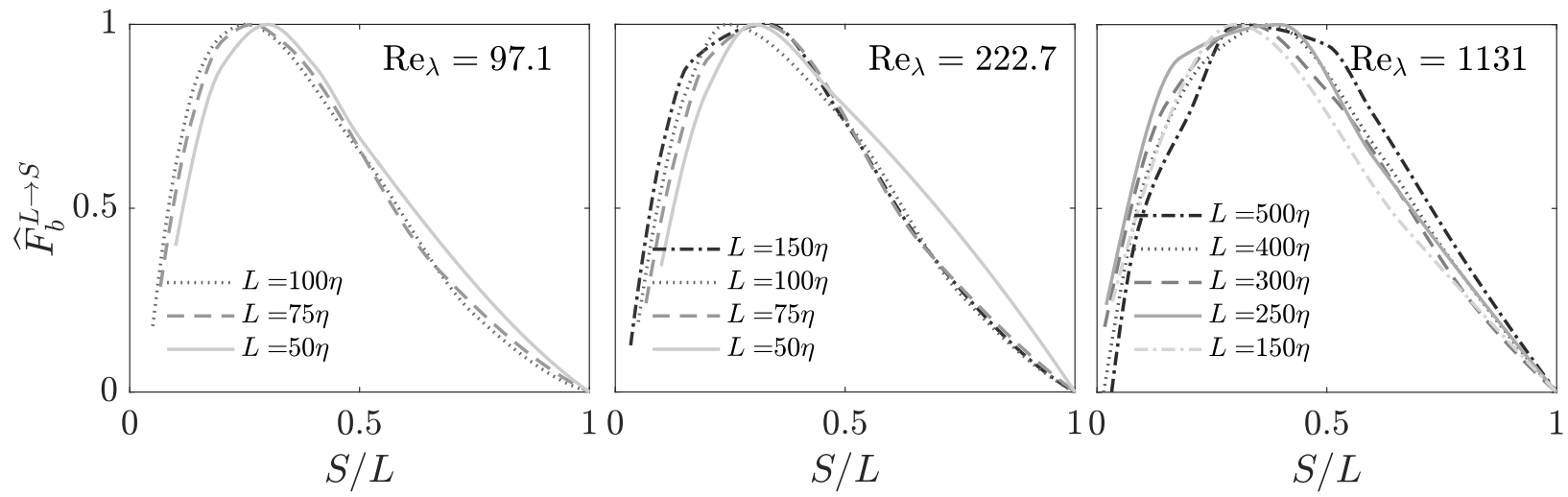

FIG. 5. Normalised enstrophy flux, $\widehat{F}_{b}^{L \rightarrow S}$, for various cases.

To further quantify the locality of this energy and enstrophy flux, one can plot the value of the ratio $S / L$ yielding the highest energy or enstrophy transfer for various $L$ and $R e_{\lambda}$ considered. These ratios, denoted as $(S / L)_{\max }^{E}$ for the energy transfer and $(S / L)_{\max }^{\Omega}$ for the enstrophy flux, are shown in Fig 6 with the uncertainty associated with the location of the peak. This uncertainty comes from the limited combination of the ratios $(S / L)$ computed 
for this study. Indeed, to estimate $(S / L)_{\max }^{\Omega}$ and $(S / L)_{\max }^{E}$, first a large scale, $L$, is chosen and then a finite number of small scales, $S$, are considered subsequently to compute the energy/enstrophy transfer and to determine for which $S$ the energy/enstrophy transfer is maximum for the chosen $L$. From this set of ratios $(S / L),(S / L)_{\max }^{\Omega}$ and $(S / L)_{\max }^{E}$ are determined. Thus, the uncertainty of the maximum corresponds to the interval between the two consecutive $(S / L)$ bracketing the peaks seen in Figs. 4 and 5. This interval width is shown as vertical bars in Fig. 6.

It is observed that for all cases, the values of $(S / L)_{\max }^{\Omega}$ and $(S / L)_{\max }^{E}$ lie between 0.25 and 0.4. This provides strong support for the scale-by-scale energy or enstrophy cascade throughout the range of scales of turbulence and more importantly, for all Reynolds numbers considered. The results shown in Figs. 4 and 5 have also been compared for the decaying and forced cases with $R e_{\lambda}$ of about 140 and no significant differences were observed (not shown) implying that the forcing of the turbulence at sufficiently large scale does not influence the energy or enstrophy flux through the scales. Furthermore, the similar behaviour of the results for both large and small scales in the cases with $R e_{\lambda}=140$ and $R e_{\lambda}=1131$ suggests that the forcing scheme does not influence the results unduly.
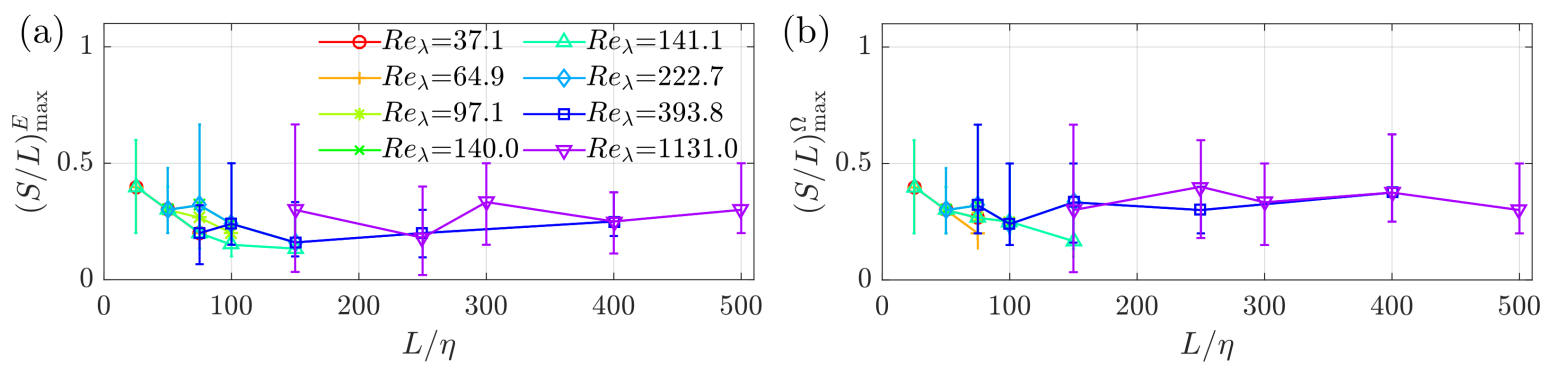

FIG. 6. Ratio (a) $(S / L)_{\max }^{E}$ and (b) $(S / L)_{\max }^{\Omega}$ yielding the maximum energy or enstrophy transfer from eddies of a scale $L$ to a scale $S$.

\section{Vorticity - strain rate alignment}

As noted by Eq. (13), the generation of enstrophy through vortex stretching mechanism is dictated by the alignment between the vorticity vector and the principal strain rates.

The probability density function of $|\cos \theta|$ between vorticity at $5 \eta$ and the principal strain rates at various $L_{s}$ is shown in Fig. 7 for four Reynolds numbers. This alignment pdf is 
shown for both the $\alpha$ and $\beta$ strain rates. The vorticity is observed to align preferentially with the most extensional strain, $\alpha$, when $L_{s}>L_{\omega}$ and the alignment with the $\beta$ strain rate increases progressively as $L_{s} / L_{\omega} \rightarrow 1$, as observed by Leung et al. [34] for $R e_{\lambda}=141$. Statistics similar to unfiltered alignment characteristics reported by Ashurst et al. [24], ie. alignment with the $\beta$ strain rate, are observed for $L_{s} \approx L_{\omega}$. This statistics with unfiltered field computed for the case $R e_{\lambda}=1131$ is shown for comparison in the frame for $L_{s}=5 \eta$ of Fig. 7 using gray lines.

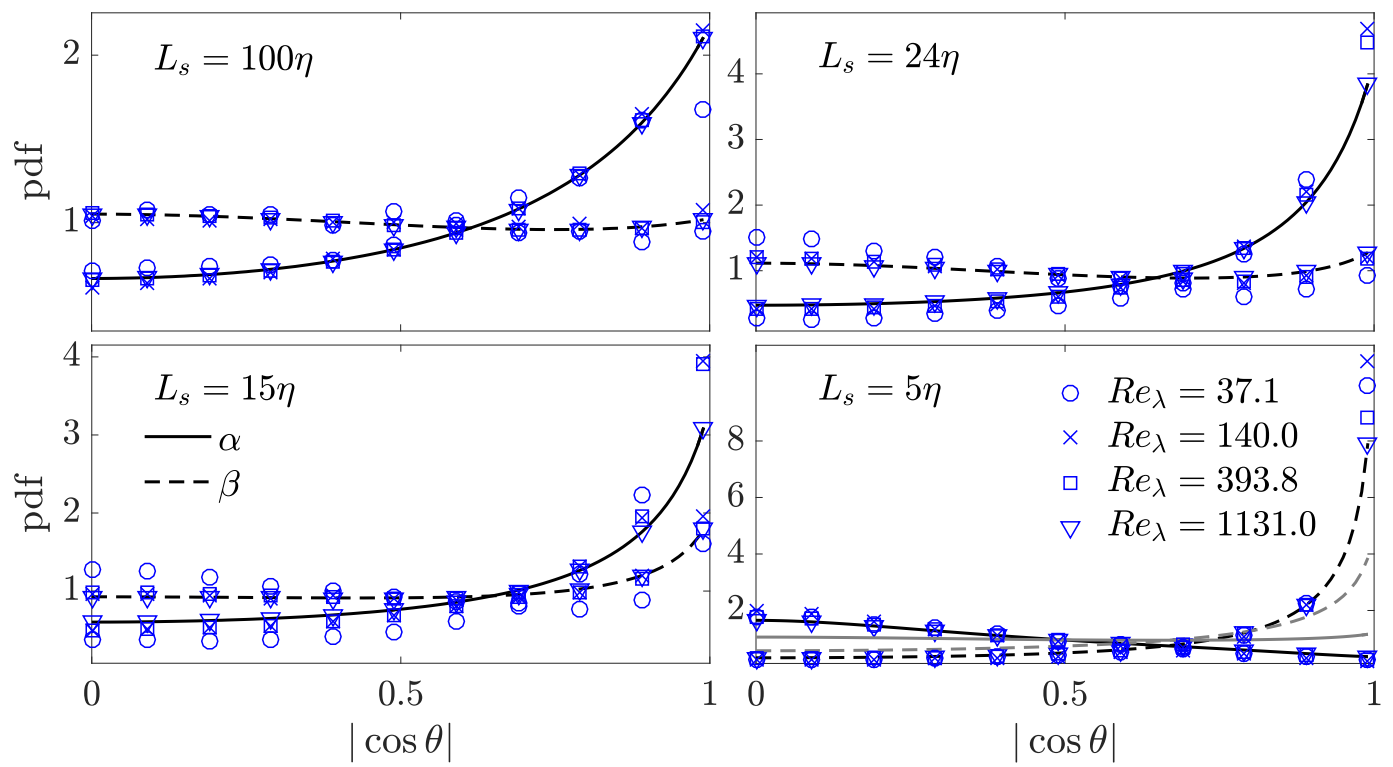

FIG. 7. Pdf of alignment between the vorticity filtered at scale $L_{\omega}=5 \eta$ and the principal directions of the strain filtered at scales $L_{s}$. Solid line $\alpha$ and dashed line $\beta$. The gray lines are for the case $R e_{\lambda}=1131$ computed using unfiltered fields.

One can also quantify the size of larger structures primarily responsible for stretching a vortex of size $L_{\omega}$ by studying the probability, $P$, of the alignment with the $\alpha$ strain rate. As discussed in Leung et al. [34], this can be obtained by simply integrating the results in Fig. 7 for $\xi_{1} \leq\left|\cos \theta_{\alpha}\right| \leq 1$ and the variation of this probability with $\mathcal{L}=L_{s} / L_{\omega}$ is shown in Fig. 8, with $\xi_{1}=0.99$, for the range of Reynolds numbers in Table I. Figures 8a and $8 \mathrm{~b}$ show this quantity respectively for $L_{\omega}=5 \eta$ and $L_{\omega}=45 \eta$ with $L_{s}$ ranging from $5 \eta$ to $1300 \eta$ depending on the dataset under analysis. (The additional case of $L_{\omega}=150 \eta$ is included for $R e_{\lambda}=1131$ in Fig. 8a). Evidently, for all cases considered, the probability peaks for $3 \leq \mathcal{L} \leq 5$, implying that the vortical structures of scale $L_{\omega}$ are stretched mostly 
by structures that are about 3 or 5 times larger, as noted by Leung et al. [34]. Although the results shown in Fig. 8 are for $\xi_{1}=0.99$, they are observed to be insensitive to the choice of the $\xi_{1}$ value. These results support the hypothesis that the enstrophy structures are predominantly stretched by the extensional strain from larger eddies, corroborating the idea that the most effective vortex stretching is incremental in scale-space. More importantly, it is seen that this observation holds for all values of $R e_{\lambda}$ investigated here and even for relatively large enstrophy structures at large $R e$ (see curve corresponding to $R e_{\lambda}=1131$ with $L_{\omega}=150 \eta$ in Fig. 8a). This observation is also consistent with the ratios of scales for the maximum energy or enstrophy transfer, $(S / L)_{\max }^{E}$ or $(S / L)_{\max }^{\Omega}$, shown in Fig 6.
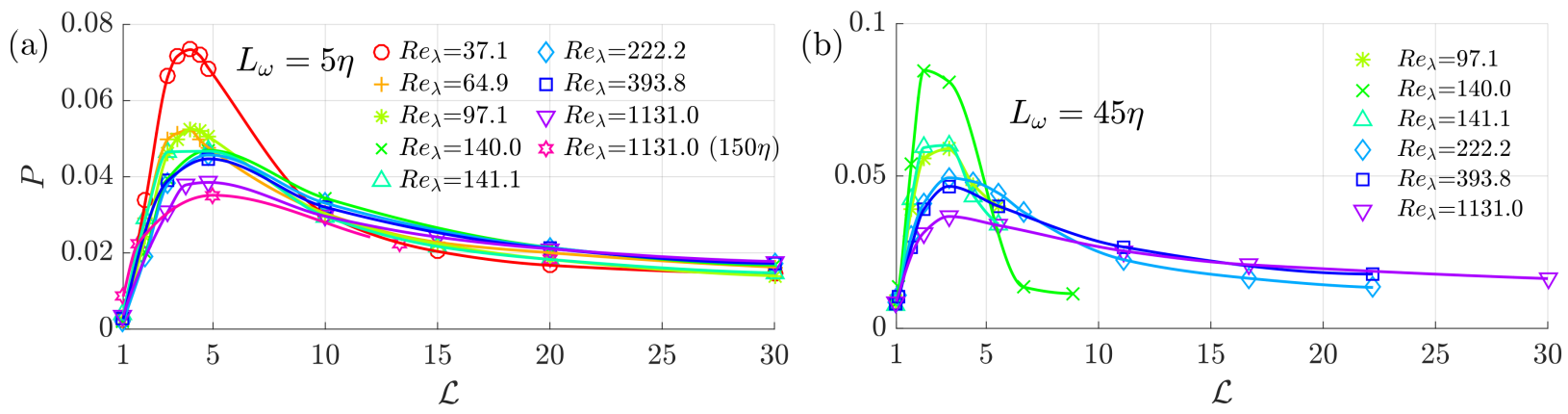

FIG. 8. Variation of probability of near perfect alignment of $\boldsymbol{\omega}$ with $\alpha$ versus $\mathcal{L}$ with (a) $L_{\omega}=5 \eta$ and (b) $L_{\omega}=45 \eta$.

The value of $\mathcal{L}$ associated with the peak probability for near perfect alignment between $\boldsymbol{\omega}$ and $\alpha$, noted as $\mathcal{L}^{*}$, is plotted against $R e_{\lambda}$ in Fig. 9 where various values of $L_{\omega}$, ranging from 5 to $100 \eta$, are considered for the strained structures. It is clear that this length scale ratio is between 3 and 5 and is more or less independent of $R e_{\lambda}$, at least for the range considered in this study and independent of the choice of $L_{\omega}$. This provides further evidence of a scaleby-scale energy transfer. The results for larger $L_{\omega}$, for example $45 \eta$, do not extend to lower $R e_{\lambda}$ because the larger straining scales required for the analysis become larger than the size of the computation domain. Furthermore, in light grayscale, the range of ratios $(L / S)_{\max }^{E}$ for each $R e_{\lambda}$ case observed in Fig. 6 is also plotted. This ratio also lies in the same range as $\mathcal{L}^{*}$ which clearly shows that all these phenomena related to the energy cascade picture are local in scale and more or less independent of the Reynolds number. This provides support and evidence for the universality of the energy cascade picture, even at modest values of $R e_{\lambda}$. 


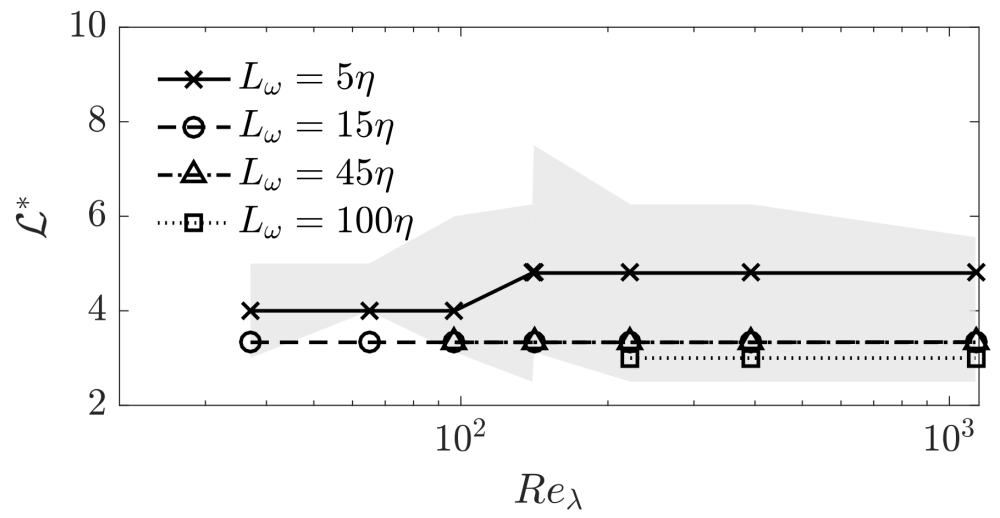

FIG. 9. Ratio $\mathcal{L}^{*}=L_{s} / L_{\omega}$ yielding the highest probability for perfect alignment of $\boldsymbol{\omega}$ with the $\alpha$ strain rate (lines). The shaded region shows the minimum and maximum values of $(L / S)_{\max }^{E}$ yielding the highest energy transfer for the associated $R e_{\lambda}$.

Furthermore, the importance of the alignment of vorticity with the $\alpha$-strain, and thus the actual vortex stretching, for the enstrophy production can be highlighted by estimating the contribution of each principal strain to the total enstrophy production. Indeed, the contributions of $\alpha, \beta$ and $\gamma$ to the volume averaged enstrophy production $\langle\psi\rangle=\left\langle\omega_{i} S_{i j} \omega_{j}\right\rangle=$ $\left\langle\psi_{\alpha}\right\rangle+\left\langle\psi_{\beta}\right\rangle+\left\langle\psi_{\gamma}\right\rangle$ is known to be in the ratio of $\left\langle\psi_{\gamma}\right\rangle:\left\langle\psi_{\beta}\right\rangle:\left\langle\psi_{\alpha}\right\rangle=-1: 1.41: 2.06$. For a vortex of scale $L_{\omega}$, these contributions from strain rates of various sizes of eddies can be estimated using

$$
\begin{aligned}
\left\langle\psi^{L_{\omega}}\right\rangle & =\left|\omega^{L_{\omega}}\right|^{2} \int_{1}^{\infty}\left(\alpha^{\mathcal{L}} \cos ^{2} \theta_{\alpha}+\beta^{\mathcal{L}} \cos ^{2} \theta_{\beta}+\gamma^{\mathcal{L}} \cos ^{2} \theta_{\gamma}\right) \mathrm{d} \mathcal{L} \\
& =\left\langle\psi_{\alpha}^{L_{\omega}}\right\rangle+\left\langle\psi_{\beta}^{L_{\omega}}\right\rangle+\left\langle\psi_{\gamma}^{L_{\omega}}\right\rangle
\end{aligned}
$$

The ratio $\left\langle\psi_{\gamma}^{L_{\omega}}\right\rangle:\left\langle\psi_{\beta}^{L_{\omega}}\right\rangle:\left\langle\psi_{\alpha}^{L_{\omega}}\right\rangle=-1: 0.46: 3.44$ is observed for $L_{\omega}=5 \eta$ and $\operatorname{Re}_{\lambda}=140$. If one considers $L_{\omega}=24 \eta$ then this ratio becomes $-1: 0.49: 4.93$. For $R e_{\lambda}=1131$, this ratio is $-1: 0.32: 2.51$ when $L_{\omega}=5 \eta$ and $-1: 0.36: 2.67$ when $L_{\omega}=150 \eta$. The $\alpha$ strain rate thus clearly dominates over the $\beta$ strain for all the filtered fields. Hence, the main mechanism of enstrophy production is through the axial vortex stretching as suggested by the original energy cascade picture [1, 19, 22]. 


\section{CONCLUSION}

In summary, the validity of the Richardson/Kolmogorov energy cascade picture has been investigated in real space using DNS data for $R e_{\lambda}$ varying from about 37 to 1131 . Through the detailed study of the energy and enstrophy fluxes, it has been confirmed that there is a scale-locality in the energy and enstrophy transfer across the cascade. In particular, it was observed that turbulent structures of a scale $L$ mostly transfer energy/enstrophy to smaller structures of a size $\sim 0.3 L$. Furthermore, as it is generally thought that the energy/enstrophy transfer is made through vortex stretching, this mechanism was studied by considering the alignment statistics between vorticity at a scale $L_{\omega}$ and straining structure at a scale $L_{s}$. It was confirmed that vortical structures of a length scale $L_{\omega}$ are mostly stretched by structures 3 to 5 times larger than $L_{\omega}$ for the range of $R e_{\lambda}$ considered. This was noted by Leung et al. [34] for $R e_{\lambda}=141$, but it is seen here that this results extend to $R e_{\lambda}$ as high as 1131 and as low as 37. In particular, the statistics of near perfect alignment between the filtered vorticity and filtered strain rate fields shows a clear maximum for a ratio of $L_{s} / L_{\omega}$ between 3 and 5 . Considering the similarity between all these findings, a close link between the vortex stretching mechanism, energy transfer and enstrophy flux can be summarised. At a given scale $L_{\omega}$, vortical structures are mostly stretched by structures of a scale of about $3 L_{\omega}$. This gives rise to a transfer of energy from this large scale $\sim 3 L_{\omega}$ to this scale $L_{\omega}$. Subsequently, at this scale $L_{\omega}$, enstrophy is being generated locally but this enstrophy is directly transferred to a smaller scale of about $0.3 L_{\omega}$ through vortex stretching at smaller scale. And this process continues down the energy cascade until the Kolmogorov and dissipative scales. Furthermore, the statistics found are qualitatively the same for all the Reynolds numbers investigated here and choice of $L_{\omega}$. This suggests the existence of a similar vortex stretching mechanism across a wide range of values of $R e_{\lambda}$ and turbulence scales and provides a strong support for the classical picture of the energy cascade and its locality. The morphology of these vortical structures has also been studied using the Minkowski functionals and were characterised as being tube-like for small scales, supporting the vortex-worm structures proposed by Burgers [21]. Thus, it is quite clear that the classical mechanism for the energy cascade prevails even at $R e_{\lambda}$ as low as about 40 or as high as 1131 .

Future work will be devoted to analysing the temporal aspect of the energy cascade. Indeed, only instantaneous snapshots were considered here while the temporal decay of the 
energy cascade could play an important role. Furthermore, the spatial locality of the energy and enstrophy transfer could also be examined in future studies.

\section{ACKNOWLEDGEMENTS}

N. A. K. Doan acknowledges the support of the Qualcomm European Research Studentship Fund in Technology. The data for $R e_{\lambda}=1131$ was provided by M. Yokokawa, T. Ishihara, K. Itakura, A. Uno and Y. Kaneda. Thanks to P. K. Yeung for providing $R e_{\lambda}=140$ data.

[1] L. F. Richardson, Weather Prediction by Numerical Process (Cambridge University Press, Cambridge, 1922).

[2] A. N. Kolmogorov, The local structure of turbulence in incompressible viscous fluid for very large Reynolds numbers, Dokl. Akad. Mak SSSR 30, 299-303 (1941).

[3] A. M. Obukhov, On the distribution of energy in the spectrum of turbulent flow, Dokl. Akad. Mak SSSR 32, 22-24 (1941).

[4] Y.-H. Pao, Structure of Turbulent Velocity and Scalar Fields at Large Wavenumbers, Phys. Fluids 8, 1063 (1965).

[5] W. Heisenberg, Zur statistischen Theorie der Kernreaktionen, Zeitschrift für Phys. 124, 628657 (1948).

[6] T. Aoyama, T. Ishihara, Y. Kaneda, M. Yokokawa, K. Itakura, and A. Uno, Statistics of energy transfer in high-resolution direct numerical simulation of turbulence in a periodic box, J. Phys. Soc. Japan 74, 3202-3212 (2005).

[7] J. A. Domaradzki and D. Carati, An analysis of the energy transfer and the locality of nonlinear interactions in turbulence, Phys. Fluids 19 (2007), 10.1063/1.2772248.

[8] G. L. Eyink and H. Aluie, Localness of energy cascade in hydrodynamic turbulence. I. smooth coarse graining, Phys. Fluids 21, 1-9 (2009), arXiv:0909.2386.

[9] H. Aluie and G. L. Eyink, Localness of energy cascade in hydrodynamic turbulence. II. Sharp spectral filter, Phys. Fluids 21, 1-16 (2009), arXiv:0909.2451. 
[10] P. A. Davidson, Turbulence : an introduction for scientists and engineers, 2nd ed. (Oxford University Press, Oxford, 2015) p. 630.

[11] M. Lesieur, Turbulence in Fluids, 4th ed. (Springer Netherlands, 2008) p. 563, arXiv:arXiv:1011.1669v3.

[12] J. A. Domaradzki, W. Liu, and M. E. Brachet, An analysis of subgrid-scale interactions in numerically simulated isotropic turbulence, Phys. Fluids A Fluid Dyn. 5, 1747 (1993).

[13] J. I. Cardesa, A. Vela-Martín, S. Dong, and J. Jiménez, The temporal evolution of the energy flux across scales in homogeneous turbulence, Phys. Fluids 27, 111702 (2015), arXiv:1505.00285.

[14] J. I. Cardesa, A. Vela-Martín, and J. Jiménez, The turbulent cascade in five dimensions, Science 7933, 1-6 (2017), arXiv:1708.00706.

[15] S. Goto, Y. Saito, and G. Kawahara, Hierarchy of antiparallel vortex tubes in spatially periodic turbulence at high Reynolds numbers, Phys. Rev. Fluids 2, 064603 (2017).

[16] J. L. Lumley, Some comments on turbulence, Phys. Fluids A Fluid Dyn. 4, 203-211 (1992).

[17] A. Tsinober, An Informal Conceptual Introduction to Turbulence (Springer Netherlands, 2009).

[18] P. K. Yeung and J. G. Brasseur, The response of isotropic turbulence to isotropic and anisotropic forcing at the large scales, Phys. Fluids A Fluid Dyn. 3, 884-897 (1991).

[19] G. I. Taylor and A. E. Green, Mechanism of the Production of Small Eddies from Large Ones, Proc. R. Soc. London A 158, 499-521 (1937).

[20] G. I. Taylor, Production and Dissipation of Vorticity in a Turbulent Fluid, Proc. R. Soc. London A 164, 15-23 (1938).

[21] J. M. Burgers, A mathematical model illustratting the theory of turbulence, Adv. Appl. Mech. 1, 171-199 (1948).

[22] R. Betchov, An inequality concerning the production of vorticity in isotropic turbulence, J. Fluid Mech. 1, 497 (1956).

[23] W. J. Cocke, Turbulent hydrodynamic line stretching: consequences of isotropy, Phys. Fluids 12, 2488-2492 (1969).

[24] Wm. T. Ashurst, A. R. Kerstein, R. M. Kerr, and C. H. Gibson, Alignment of vorticity and scalar gradient with strain rate in simulated Navier-Stokes turbulence, Phys. Fluids 30, 2343 (1987). 
[25] Z.-S. She, E. Jackson, and S. A. Orszag, Structure and dynamics of homogeneous turbulence: models and simulations, Proc. R. Soc. London A 434, 101-124 (1991).

[26] A. Vincent and M. Meneguzzi, The dynamics of vorticity tubes in homogeneous turbulence, J. Fluid Mech. 258, 245 (1994).

[27] K. Yoshimatsu, K. Anayama, and Y. Kaneda, Influence of vortex dynamics and structure on turbulence statistics at large scales, Phys. Fluids 27, 055106 (2015).

[28] L. K. Su and W. J. A. Dahm, Scalar imaging velocimetry measurements of the velocity gradient tensor field in turbulent flows. I. Assessment of errors, Phys. Fluids 8, 1883 (1996).

[29] J. A. Mullin and W. J. A. Dahm, Dual-plane stereo particle image velocimetry measurements of velocity gradient tensor fields in turbulent shear flow. II. Experimental results, Phys. Fluids 18 (2006), 10.1063/1.2166448.

[30] J. Jiménez, Kinematic alignment effects in turbulent flows, Phys. Fluids 4, 652 (1992).

[31] H. K. Moffatt, S. Kida, and K. Ohkitani, Stretched vortices - the sinews of turbulence; large-Reynolds-number asymptotics, J. Fluid Mech. 259, 241-264 (1994).

[32] P. E. Hamlington, J. Schumacher, and W. J. A. Dahm, Local and Nonlocal Strain Rate Fields and Vorticity Alignment in Turbulent Flows, Phys. Rev. E 77, 026303 (2008), arXiv:0801.1248.

[33] P. E. Hamlington, J. Schumacher, and W. J. A. Dahm, Direct assessment of vorticity alignment with local and nonlocal strain rates in turbulent flows, Phys. Fluids 20, 1-5 (2008), arXiv:0810.3439.

[34] T. Leung, N. Swaminathan, and P. A. Davidson, Geometry and interaction of structures in homogeneous isotropic turbulence, J. Fluid Mech. 710, 453-481 (2012).

[35] A. Lozano-Durán, M. Holzner, and J. Jiménez, Multiscale analysis of the topological invariants in the logarithmic region of turbulent channels at a friction Reynolds number of 932, J. Fluid Mech. 803, 356-394 (2016), arXiv:1709.00823.

[36] L. Wei, G. E. Elsinga, G. Brethouwer, P. Schlatter, and A. V. Johansson, Universality and scaling phenomenology of small-scale turbulence in wall-bounded flows, Phys. Fluids 26, 1-13 (2014).

[37] G. E. Elsinga and I. Marusic, The anisotropic structure of turbulence and its energy spectrum, Phys. Fluids 28, 1-8 (2016).

[38] D. Tordella, S. Di Savino, and L. Sitzia, Large fluctuations of the nonlinearities in isotropic turbulence. Anisotropic filtering analysis, Phys. D Nonlinear Phenom. 284, 16-26 (2014). 
[39] T. Ishihara, Y. Kaneda, and J. C. R. Hunt, Thin Shear Layers in High Reynolds Number TurbulenceDNS Results, Flow, Turbul. Combust. 91, 895-929 (2013).

[40] U. Frisch, Turbulence : the legacy of A.N. Kolmogorov (Cambridge University Press, 1995) p. 312.

[41] P. A. Davidson, K. Morishita, and Y. Kaneda, On the generation and flux of enstrophy in isotropic turbulence, J. Turbul. 9, 1-26 (2008).

[42] K. R. Mecke, T. Buchert, and H. Wagner, Robust morphological measures for large-scales structures in the universe, Astron. Astrophys. 288, 697-704 (1994).

[43] V. Sahni, B. S. Sathyaprakash, and S. F. Shandarin, Shapefinders: A New Shape Diagnostic for Large-Scale Structure, Astrophys. J. 495, L5-L8 (1998), arXiv:9801053 [astro-ph].

[44] J. V. Sheth and V. Sahni, Exploring the geometry, topology and morphology of large scale structure using Minkowski functionals (2005).

[45] M. Tanahashi, M. Myauchi, and J. Ikeda, Identification of coherent fine scale structure, in Simul. Identif. Organ. Struct. Flows, Fluid Mech. its Appl. IUTAM Symp. (1999) pp. 131-140.

[46] D. A. Donzis, P. K. Yeung, and K. R. Sreenivasan, Dissipation and enstrophy in isotropic turbulence: Resolution effects and scaling in direct numerical simulations, Phys. Fluids 20, $1-16(2008)$.

[47] H. Kobayashi, E. Matsumoto, N. Fukushima, M. Tanahashi, and T. Miyauchi, Statistical properties of the local structure of homogeneous isotropic turbulence and turbulent channel flows, J. Turbul. 12, N11 (2011).

[48] V. Eswaran and S. B. Pope, An examination of forcing in direct numerical simulations of turbulence, Comput. Fluids 16, 257-278 (1988).

[49] F. Moisy and J. Jiménez, Geometry and clustering of intense structures in isotropic turbulence, J. Fluid Mech. 513, 111-133 (2004). 